\title{
Factores de riesgo asociados con la restricción del crecimiento intrauterino.
}

\author{
Risk factors associated with the restraint of intrauterine growth.
}

\section{Correspondencia:}

Viviana Mariel Uribe-Godoy

Número de celular: 999039694

Correo Electrónico:

vivianaug@hotmail.com

Domicilio: Calle Tacones 319 San Isidro - Ica - Perú.

Contribuciones De Autoría:

VMUG, JCH : Contribuciones en la concepción y diseño del manuscrito, recolección, análisis e interpretación de los datos, redacción y revisión crítica del contenido del manuscrito y aprobación final del artículo.

Conflicto De Intereses: No declarados.

Financiamiento: Autofinanciado.

\section{Como Citar}

Uribe-Godoy Viviana Mariel. Factores de riesgo asociados con la restricción del crecimiento intrauterino. Rev méd panacea 2018; (3): 93-96

Recibido: 26 - 09 - 2018

Aceptado: 30 - 11 - 2018

Publicado: 28 - 12 - 2018

\section{RESUMEN}

Introducción: Las consecuencias de un producto con RCIU va más allá que solo el peso, sino que esta trae un alto grado de morbimortalidad tanto a mediano y a largo plazo, generando cambios en la función cardiovascular, endocrinológica y metabólica con el consiguiente aumento del riesgo de dislipidemia, hipertensión y diabetes mellitus tipo 2. Conocer sobre los factores de riesgo, permite actuar tempranamente, y así evitar consecuencias que puede tener en el neurodesarrollo infantil y en el desarrollo psicomotor. Objetivo: Realizar una búsqueda bibliográfica para determinar los factores asociados con la restricción del crecimiento intrauterino. Materiales y métodos: Es un estudio descriptivo en donde se realiza la búsqueda bibliográfica en la base de datos de Pubmed, Medline y Scielo. Discusión: La restricción del crecimiento fetal es una de las causas más comunes de peores resultados perinatales y a largo plazo, entre los factores que han descrito diversos autores, mencionan que existen riesgos ambientales, factores genéticos, factores nutriciones, factores sociodemográficos y enfermedades asociadas durante el embarazo. Conclusiones: Identificar los factores que condicionan la restricción del crecimiento intrauterino permitirá establecer el tamizaje precoz y diagnóstico oportuno contribuyendo de esta manera a la reducción de la morbimortalidad neonatal, infantil y de la población en general.

Palabras clave: Retardo del Crecimiento fetal, factores de riesgo. (fuente: DeCS BIREME).

\begin{abstract}
Introducción: The consequences of a product with IUGR goes beyond weight, but it brings a high degree of morbidity and mortality in the medium and long term, generating changes in cardiovascular, endocrinological and metabolic functions with the consequent increase in risk of dyslipidemia, hypertension and diabetes mellitus type 2 . Knowing about the risk factors, allows to act early, and thus avoid consequences that may have on child neurodevelopment and psychomotor development. Objective: To conduct a literature search to determine the factors associated with intrauterine growth restriction. Materials and methods: It is a descriptive study where the bibliographic search is carried out in the Pubmed, Medline and Scielo database. Discussion: The restriction of fetal growth is one of the most common causes of worse perinatal and long-term results, among the factors that have been described by several authors, mention that there are environmental risks, genetic factors, factors, nutrition, sociodemographic factors and associated diseases during the pregnancy. Conclusions: Identifying the factors that condition intrauterine growth restriction will allow the establishment of early screening and timely diagnosis, thus contributing to the reduction of neonatal, infant and population morbidity and mortality in general.
\end{abstract}

Keywords: Fetal Growth Retardation, risk factors. (source : MeSH NLM) 


\section{INTRODUCCIÓN}

Actualmente se ha visto que las consecuencias de un producto con RCIU va más allá que solo el peso, sino que esta trae un alto grado de morbimortalidad tanto a mediano y a largo plazo, generando cambios en la función cardiovascular, endocrinológica y metabólica con el consiguiente aumento del riesgo de dislipidemia, hipertensión y diabetes mellitus tipo 2. Además determina consecuencias en el neurodesarrollo infantil y se ha evidenciado diferencias significativas en el desarrollo psicomotor, resultados de pruebas cognitivas y comportamiento (1).

La mortalidad neonatal aumenta significativamente entre los recién nacidos con peso inferior al tercer percentil en comparación con aquellos que crecieron normalmente, además tienen cuatro a seis veces más probabilidades de sufrir parálisis cerebral1; por lo que un adecuado diagnóstico y vigilancia antenatal son fundamentales para disminuir su impacto perinatal a mediano y largo plazo (2).

Lamentablemente, la restricción del crecimiento intrauterino es una patología que no tiene tratamiento, su detección tiene por finalidad evitar complicaciones, disminuir la morbimortalidad neonatal y minimizar afecciones futuras (3). El objetivo de este artículo es realizar una revisión actualizada sobre factores prenatales asociados con la restricción del crecimiento intrauterino.

\section{MATERIALES Y MÉTODOS}

La búsqueda bibliografía se ha realizado en Pubmed, Medline y Scielo. Como criterios de selección se tomó en cuenta información publicada en dichas fuentes dada la confiabilidad de las mismas, con una antigüedad no mayor de 5 años, tanto en idioma español como inglés.

\section{RESULTADOS:}

Se encontró un total de seis artículos, siendo tres de ellos realizados en población latinoamericana, dentro de ellos un estudio peruano.

Sharma y col. (India, 2012) (4) realizo un estudio para determinar las frecuencias de los alelos polimórficos de los genes GSTM1 y GSTT1 en mujeres con restricción del crecimiento intrauterino idiopática; analizar los niveles maternos y de sangre del cordón umbilical de los pesticidas organoclorados; así como identificar el interacción del entorno genético que aumenta el riesgo de restricción del crecimiento intrauterino. Cuando se determinó la interacción entre el polimorfismo de los genes GSTM1/GSTT1 y el peso al nacer (interacción gen-ambiente), se observó una asociación significativa entre el genotipo $\beta-\mathrm{HCH}$ y GSTM1 con una reducción en el peso al nacer de $213 \mathrm{~g}$. Los autores concluyen que los niveles más altos de OCP en mujeres embarazadas se pueden considerar como un factor etiológico importante en los RGE "idiopáticos"; asimismo, el polimorfismo GST puede influir en la relación entre la exposición prenatal a los pesticidas y la restricción del crecimiento intrauterino.

Hillman y col. (Londres, 2013) (5), investigaron si la resistencia paterna a la insulina y los factores de riesgo cardiovasculares eran evidentes en el momento en que nacieron con restricción del crecimiento fetal. En los resultados se observó que los padres de niños con restricción de crecimiento fetal tenían más probabilidades de tener resistencia a la insulina, hipertensión, adiposidad central y disfunción endotelial y fumar cigarrillos en comparación con los padres de niños normales. Los autores concluyen que los hombres que tuvieron recientemente descendencia con restricción del crecimiento intrauterino tienen evidencia preclínica del síndrome de resistencia a la insulina y son más propensos a fumar que los padres de descendencia normal. El estilo de vida paterno puede influir en los factores hereditarios importantes para el crecimiento fetal.

Díaz-Granda y Díaz-Granda (Ecuador, 2015) (6), evaluaron 116 recién nacidos con RCIU hijos de adolescentes y 348 RN sin RCIU hijos de adolescentes. Los factores asociados a RCIU, según regresión logística binaria, fueron: amenaza de parto prematuro (OR 9,50; IC 95\% 4,10-22,01; $\quad \mathrm{p}=0,000)$, hipertensión arterial gestacional (OR 6,26; IC 95\% 2,37-16,51; $p=0,000$ ), sangrado gestacional (OR 2,96; IC 95\% 1,23-7,10; $\mathrm{p}=0,015)$, incremento de peso gestacional $<8 \mathrm{Kg}$ (OR 2,55; IC 95\% 1,26-5,16; $p=0,010)$, peso materno al inicio de la gestación <50 Kg (OR 2,19; IC 95\% 1,11-4,31; $\mathrm{p}=0,023$ ), controles prenatales <5 (OR 2,18; IC 95\% $1,05-4,55 ; p=0,038)$. Los autores concluyen éstos son factores maternos biológicos asociados a retardo del crecimiento intrauterino (RCIU) en hijos de adolescentes.

Verdugo-Muñoz y col. (Colombia, 2015) (7), concluyen que la ganancia inadecuada de peso, el antecedente de RCIU, la amenaza de parto pretérmino y el antecedente de cesárea fueron factores de riesgo para la restricción del crecimiento intrauterino.

Sabra y col. (España, 2017) (8) en su investigación de tipo prospectiva caso-control "Heavy metals exposure levels and their correlation with different clinical forms of fetal growth restriction" realizada para determinar si existe una asociación diferencial entre la exposición a metales pesados (cadmio, mercurio, plomo, arsénico y zinc) y la subclasificación de la pequeñez fetal en la restricción del crecimiento intrauterino (RCIU) y la edad para la edad gestacional (PEG), incluyeron 178 pares madre-hijo; 96 de peso apropiado para la edad gestacional y 82 fetos pequeños diagnosticados en el tercer trimestre. El nivel sérico fetal de cadmio $(p<0,001)$ aumentó en los fetos pequeños en comparación con los adecuados para la edad gestacional. El nivel sérico fetal de mercurio. 
$(p<0,05)$ mostró un aumento en PEG en comparación con RCIU. Solo se detectaron diferencias en los niveles entre los subgrupos de fetos pequeños en los niveles séricos fetales de cadmio y mercurio. El peso del feto se correlacionó negativamente con el nivel sérico fetal de $\mathrm{Cd}$ ( $p$ <0.001). No se observaron diferencias en los niveles de metales pesados placentarios entre los grupos. Los autores concluyen que los niveles séricos fetales de cadmio mostraron una correlación diferencial entre la subclasificación clínica de los fetos pequeños, que junto con el aumento de los niveles de cadmio en el suero tanto materno como fetal de los fetos pequeños refuerzan la influencia negativa de los metales pesados sobre el peso al nacer.

Lozano (Loreto, 2015) (9), en su tesis titulada "Factores asociados a retardo de crecimiento intrauterino en el Hospital Regional de Loreto, 2015" realizada con el objetivo de determinar los factores asociados al retardo de crecimiento intrauterino en el Hospital Regional de Loreto, los factores que se asociaron significativamente a RCIU fueron: madres menores de 20 años de edad; procedencia rural, madres con bajo peso, madres con antecedente de RN con retardo de crecimiento intrauterino, madres nulíparas $(O R=1,95 ; p=0,039)$, madres con intervalo intergenésico menor de 2 años, madres con anemia, madres con preeclampsia o eclampsia, madres con síndrome de Hellp, madres con oligohidramnios y madres con malaria. Concluye que el retardo de crecimiento intrauterino tiene asociación con factores sociodemográficos y obstétricos, que algunos de ellos pueden ser intervenidos.

\section{DISCUSIÓN:}

La restricción del crecimiento fetal es una de las causas más comunes de peores resultados perinatales y a largo plazo, y se asocia a una mayor mortalidad y morbilidad perinatal; es un problema complejo desde su diagnóstico hasta determinar el manejo prenatal y el momento óptimo para el parto (4) . Bajo estas condiciones contribuye a la mortalidad infantil, internacionalmente aceptada como un indicador clave de la condición de salud y socio-económica de una población; casi la totalidad ocurre en los países en desarrollo (10).

Entre los factores que han descrito diversos autores, mencionan que existen riesgos ambientales, factores genéticos (4), que el incremento del peso materno está en relación con el retardo de crecimiento intrauterino $(5,6)$, producto de sexo femenino, o con gemelaridad (5), el no conllevar un adecuado control prenatal (7), factores sociodemográficos y obstétricos: bajo nivel educativo, desnutrición materna, o incremento excesivo, antecedentes de partos prematuros (8), y con enfermedades asociadas, entre la principal con la hipertensión inducida en el embarazo (9).

Lamentablemente, la restricción del crecimiento intrauterino es una patología que no tiene tratamiento, su detección tiene por finalidad evitar complicaciones, disminuir la morbimortalidad neonatal y minimizar afecciones futuras. Esto se logra a través de la prevención; la misma que en este caso, debería identificar los factores asociados a la restricción de crecimiento intrauterino, como un paso obligatorio que precede a la planificación de intervenciones que reduzcan la frecuencia de desnutrición intrauterina y sus consecuencias (11).

\section{CONCLUSIÓN:}

El estudio de los factores que condicionan la restricción del crecimiento intrauterino es importe por su impacto en la salud pública -el riesgo de muerte se cuadruplica entre las 37 y 40 semanas de edad gestacional, asimismo se incrementa el riesgo de morbilidad neonatal, daño neurológico y alteración de la regulación metabólica a largo plazo- de la población. Así, la identificación de estos factores permitirá establecer características de riesgo que permitan el tamizaje precoz y diagnóstico oportuno de casos, contribuyendo de esta manera a la reducción de la morbimortalidad neonatal, infantil y de la población en general.

Un producto con retardo de crecimiento intrauterino es el índice predictivo más importante de mortalidad infantil y el factor fundamental asociado con los más de 5 millones de defunciones neonatales que ocurren cada año en el mundo. Es innegable la influencia que este tiene sobre las futuras generaciones, por lo que debe constituir el centro de los esfuerzos que el médico debe desarrollar en su prevención, sobre todo en la comunidad. Siendo un problema de Salud Pública en el Perú, no sólo por su alta morbilidad y mortalidad infantil, sino también por las secuelas que puede ocasionar en la edad adulta (hipertensión arterial, diabetes, obesidad y otros). 


\section{REFERENCIAS BIBLIOGRÁFICAS}

1. Copel JA, Ozan M. Un planteamiento práctico para la restricción del crecimiento fetal. Obstet Gynecol. 2014; 123:1057-69.

2. Sepúlveda E, Crispi F, Pons A, Gratacos E. Restricción de crecimiento intrauterino. Rev. Med. Clin. Condes. 2014; 25(6):958-963.

3. Díaz-Granda R, Díaz-Granda L. Factores fetales asociados a retardo del crecimiento intrauterino (RCIU) en hijos de adolescentes. Hospital Vicente Corral, Cuenca-Ecuador, 2013. Revista de la Facultad de Ciencias Médicas. 2017; 35(2):12-22

4. Sharma E, Mustafa M, Pathak R, Guleria K, Ahmed RS, Vaid NB, Banerjee BD. A case control study of gene environmental interaction in fetal growth restriction with special reference to organochlorine pesticides. Eur J Obstet Gynecol Reprod Biol. 2012; 161(2):163-9.

5. Hillman S, Peebles DM, Williams DJ. Paternal metabolic and cardiovascular risk factors for fetal growth restriction: a case-control study. Diabetes Care. 2013; 36(6):1675-80.

6. Verdugo-Muñoz LM, Alvarado-Llano JJ, Bastidas-Sánchez BE, Ortiz-Martínez RA. Prevalencia de restricción del crecimiento intrauterino en el Hospital Universitario San José, Popayán (Colombia), 2013. Revista Colombiana de Obstetricia y Ginecología. 2015; 66(1):46-52.
7. Díaz-Granda R, Díaz-Granda L. Factores maternos biológicos asociados a retardo del crecimiento intrauterino (RCIU) en hijos de adolescentes nacidos en el Hospital Vicente Corral, Cuenca-Ecuador. 2013. Revista Tecnológica ESPOL-RTE. 2015; 28(4):94-109.

8. Sabra S, Malmqvist E, Saborit A, Gratacós E, Gomez R. Heavy metals exposure levels and their correlation with different clinical forms of fetal growth restriction. PLoS One. 2017; 12(10):e0185645.

9. Lozano JM. Factores asociados a retardo de crecimiento intrauterino en el Hospital Regional de Loreto, 2015. Huánuco: Universidad Nacional de la Amazonía Peruana. 2017.

10. Martínez A, Pasqualini T, Bengolea V, Azaretzky M, Kuspiel F, Vaianie $E$, et al. Función gonadal en niños y adolescentes nacidos con restricción del crecimiento intrauterino. Rev Argent Endocrinol Metab. 2017; 54(4):196-203.

11. American College of Obstetricians and Gynecologists. ACOG Practice bulletin no. 134: fetal growth restriction. Obstet Gynecol. 2013; 121:1122e33.

\section{Las ediciones anteriores de revista médica PANACEA están disponibles en:}

\section{www.revpanacea.unica.edu.pe}

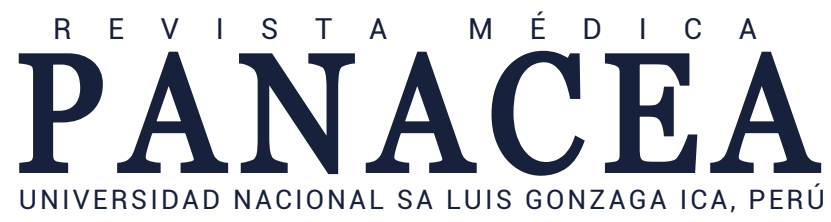

